\title{
Koordinierung von Alters- und Hinterbliebenenrenten
}

\author{
Krzysztof Ślebzak
}

$\begin{array}{lr}\text { A. Allgemeines } & 185\end{array}$

B. Versicherungsfälle $\quad 186$

C. Europäisierung der Antragstellung auf Rente 187

D. Versicherungszeiten 189

E. Berechnung der Rente 193

I. Autonome Leistung 193

II. Anteilige Leistung 193

III. Höchstbetragsregelung 194

IV. Rentenberechnung bei den Versicherungs- und Wohnzeiten
unter einem Jahr

V. Neuberechnungen und Anpassungen der Leistungen 195

F. Antikumulierungsregelungen 196

\section{A. Allgemeines}

Die Freizügigkeit der Personen innerhalb der Europäischen Union erfordert eine Koordinierung der Systeme der sozialen Sicherheit. Bei der Wahrnehmung der Freizügigkeit können die Personen den Rentensystemen mehrerer Mitgliedstaaten unterliegen. In diesem Fall sind das Europäische Parlament und der Rat verpflichtet, die notwendigen Maßnahmen für die Sicherstellung der Freizügigkeit der Arbeitnehmer vorzunehmen, insbesondere ein System einzuführen, das den zu- und abwandernden Arbeitnehmern und Selbstständigen sowie deren anspruchsberechtigten Angehörigen und den Personen, die in den Hoheitsgebieten der Mitgliedstaaten wohnen, die Anrechnung aller nach den verschiedenen innerstaatlichen Rechtsvorschriften berücksichtigten Zeiten für den Erwerb, die Berechnung und die Aufrechterhaltung des Leistungsanspruchs sichert (Art. 48 Abs. 1 lit. a) AEVV). Somit müssen die eventuellen Nachteile, die bei der Wahrnehmung der Freizügigkeit entstehen, ausgeglichen werden.

Die rechtliche Problematik von Alters- und Hinterbliebenenrenten wurde im Kapitel 5 der VO 883/2004, das das Kapitel 3 des Titels III der VO 1408/71 ersetzt, geregelt. Die Vorschriften betreffen neben den Rentenleistungen bei Alter und Tod auch die Leis- 
tungen bei Invalidität. Dies gilt jedoch nicht für Kinderzuschüsse und Waisenrenten. Gegenstand dieser Arbeit ist die Darstellung der wesentlichen Aspekte der Koordinierung der Systeme der sozialen Sicherheit bei Alter und Tod.

Was den sachlichen Geltungsbereich angeht, ist an erster Stelle zu betonen, dass dieser sich nur auf die gesetzlichen Rentensysteme bezieht. In dem sog. Dreisäulenmodell geht es somit nur um die erste Säule. Ausgenommen sind also die betrieblichen Rentensysteme (Zusatzversorgungssysteme), es sei denn, sie fallen unter den Begriff der Rechtsvorschriften gem. Art. 1 lit. 1) der VO 883/2004 oder wurden durch eine ausdrückliche Erklärung eines Mitgliedstaates einbezogen. Im polnischen Rentensystem sind somit die Offenen Pensionsfonds, bei denen der Erwerb der Leistung nicht von einer Zurücklegung der Versicherungszeiten abhängt, sondern von der Höhe des eingezahlten Rentenbeitrages, von der Koordinierung ausgeschlossen.

Zwar haben die Vorschriften des EU-Rechts vorwiegend koordinierenden Charakter, was insbesondere die Verordnungen 883/2004 (nachfolgend als Grundverordnung bezeichnet) und 987/2009 (nachfolgend als Durchführungsverordnung bezeichnet) angeht, aber es gibt auch Regelungen, die harmonisierend wirken. Vor allem im Bereich des sozialen europäischen Antidiskriminierungsrechts ist auf den allgemeinen Grundsatz der Gleichbehandlung, die aus dem AEUV abgeleiteten Antidiskriminierungsgrundsätze sowie die Richtlinien 79/7/EWG zur schrittweisen Verwirklichung des Grundsatzes der Gleichbehandlung von Männern und Frauen im Bereich der sozialen Sicherheit, 2000/43/EG zur Anwendung des Gleichbehandlungsgrundsatzes ohne Unterschied der Rasse oder der ethnischen Herkunft sowie die Richtlinie 2006/54/EG zur Verwirklichung des Grundsatzes der Chancengleichheit und Gleichbehandlung von Männern und Frauen in Arbeits- und Beschäftigungsfragen (Neufassung) hinzuweisen, wo auch die Gleichbehandlung bezüglich der Systeme der sozialen Sicherheit gefordert wird.

\section{B. Versicherungsfälle}

Versicherungsfälle sind Alter und Tod unter Zurücklassung von Ehegatten oder unterhaltsberechtigten Kindern.

Was den Versicherungsfall Alter angeht, so ist es kein natürliches Merkmal. ${ }^{1}$ Eine grundlegende Bedeutung spielt in diesem Zusammenhang die Bestimmung einer Altersgrenze, sowie die Voraussetzungen des Erwerbs eines Altersrentenanspruches. Beides wurde der Gesetzgebungskompetenz der verschiedenen Mitgliedstaaten überlassen. Ein Altersrentenanspruch besteht im Grundsatz schon ab der Vollendung des 65. Lebensjahres. Sowohl im polnischen als auch im deutschen Recht besteht jedoch auch die Möglichkeit, einen Altersrentenanspruch früher zu erwerben. Dies jedoch hängt von zusätzlichen Voraussetzungen ab (z.B. längere Versicherungszeiten, Schwerbehinde-

1 Eichenhofer, Sozialrecht der Europäischen Union, 2006, S. 130. 
rung). Ist das Alter oder die Schwerbehinderung nachzuweisen (z.B. wenn die Person in einem anderen Mitgliedstaat geboren und schwerbehindert wurde), so greift Art. 5 der Durchführungsverordnung ein, der die Rechtswirkung der in einem anderen Mitgliedstaat ausgestellten Dokumente und Belege und das Verfahren bei Zweifeln an der Gültigkeit eines Dokuments oder der Richtigkeit des Sachverhalts, der den im Dokument enthaltenen Angaben zugrunde liegt, regelt. Als Grundsatz gilt, dass vom Träger eines Mitgliedstaats ausgestellte Dokumente, in denen der Status einer Person für die Zwecke der Anwendung der Grundverordnung und der Durchführungsverordnung bescheinigt wird, sowie Belege, auf deren Grundlage die Dokumente ausgestellt wurden, für die Träger der anderen Mitgliedstaaten so lange verbindlich sind, wie sie nicht von dem Mitgliedstaat, in dem sie ausgestellt wurden, widerrufen oder für ungültig erklärt werden. Bei Zweifeln an der Gültigkeit eines Dokuments oder der Richtigkeit des Sachverhalts, der den im Dokument enthaltenen Angaben zugrunde liegt, wendet sich der Träger des Mitgliedstaates, der das Dokument erhält, an den Träger, der das Dokument ausgestellt hat, und ersucht diesen um die notwendige Klarstellung oder gegebenenfalls um den Widerruf dieses Dokuments. Der Träger, der das Dokument ausgestellt hat, überprüft die Gründe für die Ausstellung und widerruft das Dokument gegebenenfalls. Erzielen die betreffenden Träger keine Einigung, so können die zuständigen Behörden frühestens einen Monat nach dem Zeitpunkt, zu dem der Träger, der das Dokument erhalten hat, sein Ersuchen vorgebracht hat, die Verwaltungskommission anrufen.

Der zweite Versicherungsfall betrifft Tod unter Zurücklassung von Ehegatten oder unterhaltsberechtigten Kindern. Im Gegensatz zur VO 1408/71 enthält die VO 883/2004 keine Legaldefinition der Hinterbliebenen mehr. Somit wurde diese Angelegenheit den nationalen Gesetzgebern überlassen. Falls sich bei Ansprüchen auf Hinterbliebenensicherung international-familienrechtliche Vorfragen stellen, so findet an erster Stelle Art. 5 der VO 883/2004 Anwendung, der die Gleichstellung von Leistungen, Einkünften, Sachverhalten oder Ereignissen regelt. So ist auch der ausländische Familienstatus und daraus resultierende Folgen, wie ein inländischer Status, zu behandeln. Demgegenüber ist bezüglich des Nachweises bestimmter Tatsachen auf Art. 5 der Durchführungsverordnung hinzuweisen.

\section{Europäisierung der Antragstellung auf Rente}

Bei Eintritt des Versicherungsfalles ist die betreffende Person berechtigt, einen Antrag an den zuständigen Träger zu stellen. Die Wirkung von Rentenanträgen und die Rentenberechnung regelt Art. 50 VO 883/2004 in Verbindung mit Art. 45-48 der Durchführungsverordnung. Der persönliche Anwendungsbereich erstreckt sich auf alle Personen, die die Freizügigkeit wahrgenommen haben und infolgedessen hinsichtlich der oben genannten Risiken der Gesetzgebung von mindestens zwei Mitgliedstaaten 
unterlagen. Dies bedeutet, dass die betreffende Person über fremd-mitgliedstaatliche Versicherungs-, Beschäftigungs- oder Wohnzeiten verfügen muss, die für den Erwerb des Rentenanspruches Bedeutung haben. Es geht sowohl um zurückgelegte als auch um anrechnungsfähige Zeiten. Die Legaldefinition von Versicherungs-, Beschäftigungsoder Wohnzeiten beinhaltet Art. 1 lit. t), u) und v) VO 883/2004. Nach Art. 60 VO 883/2004 werden auch Personen, die einem Sondersystem für Beamte angehören, erfasst. Diese Einbeziehung entspricht auch den Vorschlägen der Kommission von 1991 und ist Folge der Berücksichtigung der Rechtsprechung des EuGH. ${ }^{2}$

Artikel 50 Abs. 1 VO 883/2004 europäisiert die rechtswirksame Antragstellung in einem Mitgliedstaat, indem die Einleitung von Leistungsfeststellungsverfahren in allen Mitgliedstaaten angeordnet wird, deren Rentenrecht für den Antragsteller gegolten hat. ${ }^{3}$ Es gibt jedoch kein einheitliches europäisches Verwaltungsverfahren. Nach Art. 45 Abs. 4 Satz 1 der Durchführungsverordnung hat der Antragsteller die Wahl, den Antrag entweder beim Träger der sozialen Sicherheit des Wohnortes oder beim Träger der letzten Beschäftigung zu stellen. Galten für die betreffende Person zu keinem Zeitpunkt die Rechtsvorschriften, die der Träger ihres Wohnorts anwendet, so leitet dieser Träger den Antrag an den Träger des Mitgliedstaats weiter, dessen Rechtsvorschriften zuletzt für sie galten. Der Zeitpunkt der Antragstellung ist für alle beteiligten Träger verbindlich (Art. 45 Abs. 4 der Durchführungsverordnung). Gibt der Antragssteller trotz Aufforderung nicht an, dass er in einem anderen Mitgliedstaat beschäftigt war oder gewohnt hat, so ist der Zeitpunkt, zu dem er seinen Antrag stellt oder zu dem er einen neuen Antrag bezüglich seiner fehlenden Beschäftigungszeiten und/oder Wohnzeiten in einem Mitgliedstaat einreicht, für den Träger, der die betreffenden Rechtsvorschriften anwendet, als Zeitpunkt der Antragsstellung, sofern diese Rechtsvorschriften keine günstigere Bestimmungen enthalten, maßgebend.

Die Antragswirkung kann jedoch auf Antrag auch begrenzt werden. Beantragt der Antragsteller nach Artikel 50 Absatz 1 der Grundverordnung, dass die Feststellung der nach den Rechtsvorschriften eines oder mehrerer Mitgliedstaaten erworbenen Altersrenten aufgeschoben wird, so hat er dies in seinem Antrag zu erklären und anzugeben, nach welchen Rechtsvorschriften er den Aufschub beantragt. Um dem Antragsteller die Ausübung dieses Rechts zu ermöglichen, teilen die beteiligten Träger ihm auf Verlangen alle ihnen vorliegenden Informationen mit, damit er die Folgen von gleichzeitigen oder nachfolgenden Feststellungen der ihm zustehenden Leistungen abschätzen kann (Art. 46 Abs. 2 der Durchführungsverordnung). Diese Vorschrift ermöglicht eine optimale Gestaltung des Rentenanspruches. In diesem Zusammenhang muss auch betont werden, dass der Aufschub der Feststellung einer Leistung nicht mit einem Leistungsverzicht gleichzusetzen ist.

Die Bearbeitung der Anträge durch die beteiligten Träger und die Mitteilung der Entscheidungen an den Antragsteller wurden in Art. 47-48 der Durchführungsverordnung

2 EuGH v. 22.11.1995, Rs. C-443/93 (Vougioukas), Slg. 1995, S. I-4033.

3 Schuler, Kommentar zum Art. 50, in: Fuchs (Hrsg.), Europäisches Sozialrecht, 2010, Rn. 6. 
geregelt. Der Kontakt-Träger übermittelt grundsätzlich die Leistungsanträge und alle ihm vorliegenden Dokumente sowie gegebenenfalls die vom Antragsteller vorgelegten einschlägigen Dokumente unverzüglich an alle beteiligten Träger. $\mathrm{Zu}$ diesem Zeitpunkt werden parallel in allen beteiligten Mitgliedstaaten Rentenfeststellungsverfahren eingeleitet. Im Laufe dieses Verfahrens können auch vorläufige Zahlungen und Vorschüsse geleistet werden, was in Art. 50 der Durchführungsverordnung geregelt ist und sowohl für eine autonome (Abs. 1) als auch für eine anteilige (Abs. 2) Leistung gilt.

Das Einreichen des Antrages bei dem Kontakt-Träger entfaltet die gleichen Folgen, wie wenn der Antrag bei dem zuständigen Träger gestellt wurde. ${ }^{4}$ Bei einem eventuellen Rechtsstreit betreffend das Datum der Antragstellung ist das bei dem ersten Träger maßgebend. ${ }^{5}$

Sollte der Leistungsanspruch nicht in allen Mitgliedsländern begründet sein, so findet Art. 50 Abs. 2 VO 883/2004 Anwendung, der das Günstigkeitsprinzip zum Ausdruck bringt. In diesem Fall lassen die Träger, nach deren Rechtsvorschriften die Voraussetzungen erfüllt sind, bei der Berechnung nach Art. 52 Abs. 1 Buchstabe a) oder b) der Durchführungsverordnung die Zeiten, die nach den Rechtsvorschriften zurückgelegt wurden, deren Voraussetzungen nicht oder nicht mehr erfüllt sind, unberücksichtigt, wenn diese Berücksichtigung $\mathrm{zu}$ einem niedrigeren Leistungsbetrag führt. Dieser Grundsatz gilt auch für den Fall, dass die Altersgrenze nicht erreicht wird (Abs. 2). „Dieser Regelung liegt die Prämisse zugrunde, dass die Leistungsberechnung nach Art. 52 Abs. 1 lit. a) und b) ohne die Berücksichtigung von Zeiten des oder der Mitgliedstaaten erfolgt, nach deren/dessen Rechtsvorschriften die Leistungsvoraussetzungen nicht erfüllt oder nicht beantragt sind, gegebenenfalls ein günstigeres pro-rata-Verhältnis ergibt oder eine Prorataisierung ganz entfällt und zu einem höheren Leistungsbetrag führen kann". 6

\section{Versicherungszeiten}

Für den Erwerb des Rentenanspruches sind die Versicherungszeiten von grundlegender Bedeutung. Darunter fallen die Beitragszeiten, Beschäftigungszeiten oder Zeiten einer selbstständigen Erwerbstätigkeit, die nach den Rechtsvorschriften, nach denen sie zurückgelegt worden sind oder als zurückgelegt gelten, als Versicherungszeiten bestimmt oder anerkannt sind, sowie alle gleichgestellten Zeiten, soweit sie nach diesen Rechtsvorschriften als den Versicherungszeiten gleichwertig anerkannt sind (Art. 1 lit. t) VO 883/2004). Somit gelten als Versicherungszeiten die als Pflicht- oder freiwil-

4 EuGH v. 9.3.1976, Rs. 108/75 (Balsamo), Slg. 1976, S. 375.

5 EuGH v. 24.10.1996, Rs. C-335/95 (Piccard), Slg. 1996, S. I-5625.

6 Schuler, Kommentar zum Art. 50 (Fußn. 3), Rn. 15. 
lig Versicherter verbrachten sowie die diesen gleichgestellten, den Ausgleich eines Rechtsverlustes bezweckenden Zeiten. ${ }^{7}$

Die rechtliche Qualifikation von Versicherungszeiten erfolgt aufgrund der inländischen Gesetzgebung. Beurteilt sich ein Sachverhalt nach dem polnischen oder nach dem deutschen Recht, kommt es auf die Beitragszeiten oder beitragslosen Zeiten an, wobei in Deutschland bei den beitragslosen Zeiten zusätzlich zwischen Anrechnungs-, Kindererziehungs- und Zurechnungszeiten differenziert wird. ${ }^{8}$ Als besonderes Problem in Deutschland stellt sich die Berücksichtigung von Kindererziehungszeiten dar. Nach dem inländischen Recht ist grundsätzlich nur in Deutschland erbrachte Erziehung als Kindererziehungszeit anzuerkennen. Ausnahmsweise kann auch die in einem anderen Mitgliedstaat erbrachte Erziehung anerkannt werden, falls sich diese unmittelbar an eine Inlandsbeschäftigung des Erziehenden anschließt oder der Ehegatte im Ausland unter deutschem Sozialrechtsstatus tätig ist. ${ }^{9}$

Die ausländischen Versicherungszeiten können aber nur dann angerechnet werden, wenn ein Versicherter in dem nach dem inländischen Recht geforderten Umfang nicht über für den Erwerb der Rente ausreichende Versicherungszeiten verfügt. Erst dann ist eine anspruchsbegründende Anrechnung von fremden Versicherungszeiten zulässig. Hat demgegenüber der Versicherte die erforderlichen Versicherungszeiten nach dem inländischen Recht erbracht, so sind die ausländischen Zeiten für die Feststellung des Erwerbs eines Rentenanspruchs unerheblich. Das Gebot der Zusammenrechnung erstreckt sich auf alle Rechtsnormen eines Mitgliedstaates, von denen der Erwerb, die Aufrechthaltung oder das Wiederaufleben des Anspruchs auf die Leistung abhängt (Art. 51 VO 883/2004).

Ein interessanter Fall wurde mit der Sache Tomaszewska ${ }^{10}$ in einem Vorabentscheidungsersuchen beim EuGH erörtert. In der Sache ging es um eine polnische Regelung, die die Berücksichtigung der beitragslosen Zeiten nur in einem bestimmten Verhältnis zu den Beitragszeiten erlaubt (ein Drittel). Die Versicherte verfügte über Beitragszeiten in der Tschechischen Republik. Darüber hinaus lagen auch polnische Beitragszeiten und beitragslose Zeiten vor. Weil in diesem Fall nach polnischem Recht für den Leistungserwerb eine 20jährige Mindestversicherungszeit vorausgesetzt war, stellte sich die Frage, ob die Zurechnung der tschechischen Versicherungszeiten nach den Koordinierungsregelungen erst nach der Berechnung der Versicherungszeiten gemäß den polnischen Regelungen zu erfolgen habe und dann addiert werden solle. Die Versicherte vertrat die Meinung, dass die Berücksichtigung von ausländischen Versicherungszeiten schon bei der Feststellung der Versicherungszeit nach dem polnischen Recht erfolgen solle. Je nach der gewählten Methode kommt man zu anderen Ergebnissen. Nach der Rechtsprechung des EuGH ist Art. 45 Abs. 1 VO 1408/71 des Rates zur Anwendung der Systeme

7 EuGH v. 3.10.2002, Rs. C-347/00 (Barreira Pérez), Slg. 2002, S. I-8191.

8 Eichenhofer, Sozialrecht (Fußn. 1), Rn. 218.

9 EuGH v. 23.11.2000, Rs. C-135/99 (Elsen), Slg. 2000, S. I-10409.

10 EuGH v. 3.3.2011, Rs. C-440/09, noch nicht in der Slg. 
der sozialen Sicherheit auf Arbeitnehmer und Selbständige sowie deren Familienangehörige, die innerhalb der Gemeinschaft zu- und abwandern, dahingehend auszulegen, dass bei der Bestimmung der nach nationalem Recht für den Erwerb des Anspruchs auf eine Altersrente durch einen Arbeitnehmer erforderlichen Mindestversicherungszeit der zuständige Träger des betreffenden Mitgliedstaats zur Bestimmung der Grenze, die die beitragsfreien Zeiten im Verhältnis zu den Beitragszeiten nicht übersteigen dürfen, wie sie in der Regelung dieses Mitgliedstaats vorgesehen ist, alle von dem Arbeitnehmer im Laufe seines Berufslebens zurückgelegten Versicherungszeiten einschließlich der in anderen Mitgliedstaaten zurückgelegten zu berücksichtigen hat.

Die Zusammenrechnung von ausländischen Zeiten beschränkt sich nur auf die Anspruchsbegründung. Für die Berechnung der Leistungshöhe sind die in anderen Mitgliedstaaten erbrachten Zeiten ohne Bedeutung. Vor polnischen Gerichten wurde diese Frage mehrmals erörtert. Der Oberste Gerichtshof stellte fest, dass im Ausland erzielte Einkommen sowie zurückgelegte Versicherungszeiten bei der Berechnung der Höhe einer Altersrente in Polen nicht berücksichtigt werden können. ${ }^{11}$ Dies gilt auch für die Berechnung des sog. Anfangskapitals für die Versicherten, die im Jahre 1999 dem neuen Altersrentensystem beigetreten sind. ${ }^{12}$ Von der Koordinierung ist im polnischen Recht die sog. zweite Säule, die kapitalgedeckt finanziert wird, ausgenommen, weil in diesem System der Leistungserwerb nicht von der Zurücklegung der Versicherungszeiten abhängt. Für den Leistungserwerb wird nur das Erreichen einer Altersgrenze sowie das Verfügen über ein aus den eingezahlten Beiträgen gebildetes Kapital vorausgesetzt.

Eine Zusammenrechnung darf auch nur dann stattfinden, wenn die Versicherungszeiten sich nicht überschneiden.

Besondere Regelungen bezüglich der Zusammenrechnung von Zeiten findet man in Art. 51 und Art. 57 VO 883/2004. Die erstgenannte Regelung betrifft die Zusammenrechnung von Versicherungszeiten, die in einem Sondersystem für beschäftigte und selbständig erwerbstätige Personen erbracht wurden. Ist nach den Rechtsvorschriften eines Mitgliedstaats die Gewährung bestimmter Leistungen davon abhängig, dass die Versicherungszeiten nur in einer bestimmten Beschäftigung oder selbstständigen Erwerbstätigkeit oder einem Beruf zurückgelegt wurden, für die ein Sondersystem für beschäftigte oder selbständig erwerbstätige Personen gilt, so berücksichtigt der zuständige Träger dieses Mitgliedstaats die nach den Rechtsvorschriften eines anderen Mitgliedstaats zurückgelegten Zeiten nur dann, wenn sie in einem entsprechenden System, oder, falls es ein solches nicht gibt, in dem gleichen Beruf oder gegebenenfalls in der gleichen Beschäftigung oder selbständigen Erwerbstätigkeit zurückgelegt wurden. Dadurch wird eine Gleichstellung bei der Berücksichtigung ausländischer Zeiten in der beschriebenen Weise angeordnet. Erfüllt jedoch die betreffende Person auch unter Berücksichtigung solcher Zeiten nicht die Anspruchsvoraussetzungen für Leistungen im Rahmen eines Sondersystems, so werden diese Zeiten für die Gewährung von Leistun-

11 Urteil des Obersten Gerichts v. 24.10.2006, Rs. II UK 98/06, OSNP 2007/21-22/332.

12 Urteil des Obersten Gerichts v. 2.9.2009, Rs. II UK 30/09, OSNP 2011/9-10/128. 
gen des allgemeinen Systems oder, falls es ein solches nicht gibt, des Systems für Arbeiter bzw. Angestellte berücksichtigt, sofern die betreffende Person dem einen oder anderen dieser Systeme angeschlossen war.

Eine besondere Regelung gilt auch für die Berücksichtigung von Minizeiten. Eine Anrechnung kommt nach Art. 57 VO 883/2004 nicht in Betracht, falls die Versicherungszeit die Dauer von zwölf Monaten nicht übersteigt.

Ein interessantes Problem bei der Zusammenrechnung von Versicherungszeiten betrifft die Frage einer kumulativen Anwendung der Vorschriften über die Anrechnung aus mehreren internationalen Koordinationsabkommen, inklusive der EU-Verordnungen. Die Rechtsprechung des EuGH gebietet die kumulative Anwendung ausdrücklich, soweit die Abkommen solch eine Möglichkeit nicht ausschließen. ${ }^{13}$

Auch für Personen, die einem gesonderten System für Beamte angehören, wurde eine spezielle Regelung bezüglich der Berücksichtigung von Versicherungszeiten vorgesehen. Das ist Ausdruck der Einbeziehung der Sondersysteme für Beamte in die Koordinierung (für diese Personen gelten Art. 6, 50, 51 Abs. 3 und Art. 52 bis 59 entsprechend). Was die Zusammenrechnung von Zeiten in einem gesonderten System für Beamte angeht, so findet Art. 60 Abs. 2 VO 883/2004 Anwendung. Ist nach den Rechtsvorschriften eines zuständigen Mitgliedstaates der Erwerb, die Auszahlung, die Aufrechterhaltung oder das Wiederaufleben des Leistungsanspruches aufgrund eines Sondersystems für Beamte davon abhängig, dass alle Versicherungszeiten in einem oder mehreren Sondersystemen für Beamte in diesem Mitgliedstaat zurückgelegt wurden oder durch die Rechtsvorschriften dieses Mitgliedstaats solchen Zeiten gleichgestellt sind, so berücksichtigt der zuständige Träger dieses Staates nur die Zeiten, die nach den für ihn geltenden Rechtsvorschriften anerkannt werden können. „Die grundsätzliche Einbeziehung der Beamten in die gemeinschaftliche Koordinierung in Abs. 1 wird hierdurch für den Bereich der Sondersysteme für Beamte wieder aufgehoben und der Regelungskompetenz der Mitgliedstaaten überantwortet. Fremdmitgliedschaftliche Zeiten sind insoweit nur dann und gegebenfalls in dem Umfang zu berücksichtigen, in welchem dies von der anzuwendenden nationalen Rechtsvorschriften vorgesehen. " 14 Erfüllt die betreffende Person auch unter Berücksichtigung solcher Zeiten nicht die Voraussetzungen für den Bezug dieser Leistungen, so werden diese Zeiten für die Feststellung von dem allgemeinen System oder, falls es ein solches nicht gibt, im System für Arbeiter bzw. Angestellte berücksichtigt.

13 EuGH v. 15.1.2002, Rs. C-55/00 (Gottardo), Slg. 2002, S. I-413.

14 Schuler, Kommentar zum Art. 60 (Fußn. 3), Rn. 56. 


\section{E. Berechnung der Rente}

Die Berechnung der Rente ist in Art. 52 VO 883/2004 normiert. Es sind zwei Möglichkeiten der Berechnung vorgesehen, je nachdem, ob nur die nationale (autonome Leistung) oder internationale (anteilige Leistung) Berücksichtigung von Versicherungszeiten in Betracht kommt. An dieser Stelle muss betont werden, dass es im europäischen Koordinierungsrecht nicht zu einer eigenständigen Rentenberechnung kommt.

\section{Autonome Leistung}

Die autonome Leistung wird dann berechnet, wenn die Voraussetzungen für den Leistungsanspruch ausschließlich nach nationalem Recht erfüllt wurden (Art. 52 Abs. 1 lit. a)). Erfüllt der Versicherte die Voraussetzungen für den Erwerb einer Rente nur nach dem inländischen Recht, so muss zusätzlich eine Vergleichsberechnung der festgestellten autonomen Leistung mit der anteiligen Leistung angestellt werden. Nach Maßgabe des Art. 52 Abs. 3 VO 883/2004 steht dem Versicherten gegenüber dem Träger jedes Mitgliedstaates die höhere (Teil-)Rente zu.

Zugleich sieht Abs. 4 die Möglichkeit vor, auf den Vergleich zu verzichten, wenn die Berechnung der autonomen Leistung dazu führt, dass diese Leistung gleich hoch wie oder höher als die anteilige Leistung ist. ${ }^{15}$ Ist der Rentenanspruch nur unter Berücksichtigung fremdmitgliedstaatlicher Zeiten erfüllt, so erfolgt die Berechnung der anteiligen Leistung.

\section{Anteilige Leistung}

Um die Nachteile der Wahrnehmung der Freizügigkeit zu vermeiden, wurde die Möglichkeit der Berechnung einer anteiligen Leistung eingeführt. Dies gilt vor allem in allen Fällen, wo die Berücksichtigung von ausländischen Versicherungszeiten für den Erwerb des Rentenanspruches notwendig ist. „In dem ersten Schritt wird ein theoretischer Rentenbetrag errechnet und dabei (jeweils) eine rein innerstaatliche Rentenbiografie unterstellt, d.h. alle fremdmitgliedstaatlichen Zeiten sind zu berücksichtigen, als ob sie nach inländischen Recht zurückgelegte anrechnungsfähige und damit auch abzugeltende Zeiten darstellen. In einem zweiten Schritt wird aus dieser so ermittelten hypothetischen Gesamtrente, dem theoretischen Betrag, nach dem Verhältnis der in den Mitgliedstaaten vor Eintritt des Versicherungsfalles zurückgelegten Versicherungszeiten der tatsächlich zu zahlende Betrag, der ,tatsächliche Betrag der anteiligen Leistung

15 EuGH v. 15.12.1993, Rs. C-113/92, C-114/92 und C-156/92 (Fabrizii, Neri und Del Grosso), Slg. 1993, S. I-6707. 
errechnet." 16 Dies ergibt sich ausdrücklich aus Art. 52 Abs. 1 lit. b) VO 883/2004. Der theoretische Betrag der Leistung entspricht der Leistung, auf die die betreffende Person Anspruch hätte, wenn alle nach den Rechtsvorschriften der anderen Mitgliedstaaten zurückgelegten Versicherungs- und/oder Wohnzeiten nach den für diesen Träger zum Zeitpunkt der Feststellung der Leistung geltenden Rechtsvorschriften zurückgelegt worden wären. Ist nach diesen Rechtsvorschriften die Höhe der Leistung von der Dauer der zurückgelegten Zeiten unabhängig, so gilt dieser Betrag als theoretischer Betrag. Der zuständige Träger ermittelt sodann den tatsächlichen Betrag der anteiligen Leistung auf der Grundlage des theoretischen Betrags nach dem Verhältnis zwischen den gesamten nach den Rechtsvorschriften aller beteiligten Mitgliedstaaten vor Eintritt des Versicherungsfalls zurückgelegten Zeiten.

Eine ergänzende Vorschrift für die Berechnung der anteiligen Leistung beinhaltet Art. 56 VO 883/2004, der zugleich eine besondere Methode der Rentenberechnung für den Fall einer vorgeschriebenen Höchstdauer für Versicherungs- und Wohnzeiten (Abs. 1 lit. a) und b)) und einer Leistungsberechnung nach einem Durchschnittsentgelt, einem Durchschnittsarbeitseinkommen (Abs. 1 lit. c)) vorsieht.

Aus der Rechtsprechung des EuGH ergibt sich, dass die Berücksichtigung der fremdmitgliedstaatlichen Versicherungszeiten auch dann geboten ist, wenn diese Zeiten nach dem inländischen Recht des zuständigen Trägers nicht berücksichtigt werden müssten. ${ }^{17}$ Zugleich ist den Mitgliedstaaten nicht erlaubt, Vorschriften zu erlassen, durch die die Berechnung des theoretischen Betrages gegenüber demjenigen nach den allgemeinen Vorschriften herabgesetzt wird. 18

Was den Umfang der zu berücksichtigenden Versicherungszeiten bei der Anwendung der inländischen Methode der Berechnung einer Altersrente angeht, findet Art. 51 VO 883/2004 entsprechend Anwendung. In der Praxis ist es oft schwierig, die ausländischen Zeiten bei den einzelnen Berechnungsfaktoren zu berücksichtigen, insbesondere im Fall der Bewertung beitragsfreier Zeiten. Im deutschen Recht erfolgt die Bewertung von beitragsfreien Zeiten nach dem sog. Gesamtleistungsmodell. Das bedeutet, dass Monate, die nicht mit Beiträgen belegt sind, die „Belegungsdichte“ vermindern und die Bewertung negativ beeinflussen. 19

\section{Höchstbetragsregelung}

Gemäß Art. 52 Abs. 3 hat die betreffende Person gegenüber dem zuständigen Träger jedes Mitgliedstaats Anspruch auf den höheren der Leistungsbeträge, die nach Absatz 1

16 Schuler, Kommentar zum Art. 52 (Fußn. 3), Rn. 15.

17 EuGH v. 15.12.1993, Rs. C-113/92, C-114/92 und C-156/92 (Fabrizii, Neri und Del Grosso) (Fußn. $15)$.

18 EuGH v. 23.9.1982, Rs. C-274/81 (Basem), Slg. 1982, S. 2995.

19 Schuler, Kommentar zum Art. 52 (Fußn. 3), Rn. 24. 
Buchstaben a) und b) berechnet wurden. Somit wurde auch in der VO 883/2004 auf die Begrenzung des theoretischen Betrages verzichtet. In dieser Regelung kommt das Günstigkeitsprinzip zum Ausdruck, das unmittelbar aus dem EU-Recht und der Rechtsprechung des EuGH zu entnehmen ist. ${ }^{20}$ Dieser Grundsatz gilt nicht, wenn eine Person während desselben Zeitraumes in zwei Mitgliedstaaten beschäftigt war und während dieses Zeitraumes in beiden Mitgliedstaaten Beiträge zur Altersrentenversicherung gezahlt hat. ${ }^{21}$

\section{Rentenberechnung bei den Versicherungs- und Wohnzeiten unter einem Jahr}

An erster Stelle ist darauf hinzuweisen, dass für die Zwecke der Anwendung des Art. 57 VO 883/2004 der Begriff der „Zeiten“ definiert wurde. Dieser Ausdruck bezeichnet alle Versicherungszeiten, Beschäftigungszeiten, Zeiten einer selbständigen Erwerbstätigkeit oder Wohnzeiten, die entweder für den Leistungsanspruch oder unmittelbar für die Leistungshöhe heranzuziehen sind. Aus Gründen der Verwaltungsvereinfachung und zur Vermeidung der Auszahlung sehr geringer Renten, ist der Träger eines Mitgliedstaates nicht verpflichtet, Leistungen für Zeiten zu gewähren, die nach den für ihn geltenden Vorschriften zurückgelegt wurden und bei Eintritt des Versicherungsfalls zu berücksichtigen sind, wenn die Dauer dieser Zeiten weniger als ein Jahr beträgt und aufgrund allein dieser Zeiten kein Leistungsanspruch nach diesen Rechtsvorschriften erworben wurde. Es ist damit eine autonome Leistung gemeint. Folge der Leistungsfreistellung ist, dass die fraglichen Zeiten von dem zuständigen Träger jedes anderen Mitgliedstaates bei der Ermittlung des theoretischen Betrages nach Art. 52 Abs. 1 lit. b) zu berücksichtigen sind. Diese Pflicht besteht unabhängig davon, ob der Versicherte in dem berücksichtigenden Mitgliedstaat einen Anspruch auf die autonome Leistung erwerben kann. ${ }^{22}$ Bei einem Zusammentreffen von Minizeiten (Abs. 3) wird der zuständige Träger des Mitgliedstaates, nach dessen Rechtsvorschriften zuletzt vor Eintritt des Versicherungsfalles Versicherungs- oder Wohnzeiten zurückgelegt wurden und dessen Leistungsvoraussetzungen erfüllt sind, zur Leistung unter Berücksichtigung aller übrigen mitgliedstaatlichen Versicherungs- und Wohnzeiten verpflichtet.

\section{$V$. Neuberechnungen und Anpassungen der Leistungen}

Die rechtliche Problematik der Neuberechnung und Anpassung der Leistungen wurde in Art. 59 VO 883/2004 geregelt. Die aktuelle Fassung des Absatzes 1 (Neuberech-

20 EuGH v. 21.10.1975, Rs. C-24/75 (Petroni), Slg. 1975, S. 0149; EuGH v. 13.10.1977, Rs. C-112/76 (Manzoni), Slg. 1977, S. 1647.

21 EuGH v. 2.8.1993, Rs. C-31/92 (Larsy), Slg. 1993, S. I-4543.

22 EuGH v. 18.2.1982, Rs. C-55/81 (Vertnant), Slg. 1982, S. 649. 
nung) ist auf die bisherige Rechtsprechung des EuGH zurückzuführen. Insbesondere geht es um die Sache Bogana ${ }^{23}$. Darüber hinaus werden die Leistungen neu berechnet, wenn es zu einer Änderung des Feststellungsverfahrens oder zu einer Änderung der Berechnungsmethode nach inländischem Recht gekommen ist. Nach der Rechtsprechung des EuGH ist die Neuberechnung auch dann vorzunehmen, wenn es zu einer Änderung der Berechnungsfaktoren und der Um- und Rückverwandlungen von Hinterbliebenenrenten und Renten kommt. ${ }^{24}$

Die Anpassung der Renten wurde demgegenüber in Abs. 2 geregelt. Der Prozentsatz oder der Betrag, um den bei einem Anstieg der Lebenshaltungskosten, bei Änderung des Lohnniveaus oder aus anderen Anpassungsgründen die Leistungen des betreffenden Mitgliedstaates geändert werden, gilt unmittelbar für die nach Art. 52 festgestellten Leistungen, ohne dass eine Neuberechnung vorzunehmen ist. Absatz 2 findet jedoch auf Leistungen wie die des garantierten Altersmindesteinkommens keine Anwendung. ${ }^{25}$

\section{F. Antikumulierungsregelungen}

Um ein Zusammentreffen der inländischen Antikumulierungsregelungen bei der Berechnung der Altersrenten zu vermeiden, wurden in der VO 883/2004 besondere Vorschriften eingeführt, die die Grenzen der Antikumulierung festsetzen (Art. 53-55). Artikel 53 VO 883/2004 beinhaltet die Legaldefinitionen des Zusammentreffens von Leistungen gleicher Art (Abs.1) und unterschiedlicher Art (Abs. 2) und trifft einheitliche Koordinierungsregelungen für das Zusammentreffen dieser Leistungen (Abs. 3). Jedes Zusammentreffen von Leistungen bei Invalidität, bei Alter oder an Hinterbliebene, die auf der Grundlage der von derselben Person zurückgelegten Versicherungs- und/oder Wohnzeiten berechnet oder gewährt wurden, gilt als Zusammentreffen von Leistungen gleicher Art. Nach der Rechtsprechung des EuGH gelten Leistungen der sozialen Sicherheit nur dann als Leistungen gleicher Art, wenn ihr Gegenstand und ihr Zweck sowie ihre Berechnungsgrundlage und die Voraussetzungen für die Gewährung identisch sind. ${ }^{26}$ Demgegenüber gilt das Zusammentreffen von Leistungen, die nicht als Leistungen gleicher Art im Sinne des Absatzes 1 angesehen werden, als Zusammentreffen von Leistungen unterschiedlicher Art.

Der zuständige Träger berücksichtigt die in einem anderen Mitgliedstaat erworbenen Leistungen oder erzielten Einkünfte nur dann, wenn die für ihn geltenden Rechtsvorschriften Berücksichtigung von im Ausland erworbenen Leistungen oder erzielten Ein-

23 EuGH v. 18.2.1993, Rs. C-193/92 (Bogana), Slg. 1993, S. I-755.

24 EuGH v. 17.5.1984, Rs. C-104/83 (Cinciulo), Slg. 1984, S. 1285.

25 EuGH v. 22.4.1993, Rs. C-65/92 (Levationo), Slg. 1993, S. I-2005.

26 EuGH v. 30.9.1997, Rs. C-36/96 (Cordelle), Slg. 1998, S. I-583; v. 7.3.2002, Rs. C-107/00 (Insalaca), Slg. 2002, S. I-2403. 
künften vorsehen (Abs. 3 lit. a)). In diesem Fall sind die Mitgliedstaaten verpflichtet, dies entsprechend zu regeln. ${ }^{27}$

Der zuständige Träger berücksichtigt nach den in der Durchführungsverordnung festgelegten Bedingungen und Verfahren den von einem anderen Mitgliedstaat zu zahlenden Leistungsbetrag vor Abzug von Steuern, Sozialversicherungsbeiträgen und anderen individuellen Abgaben oder Abzügen, sofern nicht die für ihn geltenden Rechtsvorschriften vorsehen, dass die Doppelleistungsbestimmungen nach den entsprechenden Abzügen anzuwenden sind (Abs. 3 lit. b)).

Nach Abs. 4 lit. c) ist die Berücksichtigung ausländischer Leistungen ausgeschlossen, soweit diese auf der Grundlage einer freiwilligen (Weiter-)Versicherung gewährt werden.

Wendet ein einzelner Mitgliedstaat Doppelleistungsbestimmungen an, weil die betreffende Person Leistungen gleicher oder unterschiedlicher Art nach den Rechtsvorschriften anderer Mitgliedstaaten bezieht oder in anderen Mitgliedstaaten Einkünfte erzielt hat, so kann die geschuldete Leistung nur um den Betrag dieser Leistungen oder Einkünfte gekürzt werden (Abs. 3 lit. d)).

Das Zusammentreffen von Leistungen gleicher Art wurde in Art. 54 VO 883/2004 geregelt. Der Wortlaut knüpft an die Rechtsprechung des EuGH an. ${ }^{28}$ Treffen Leistungen gleicher Art, die nach den Rechtsvorschriften von zwei oder mehr Mitgliedstaaten geschuldet werden, zusammen, so gelten die in den Rechtsvorschriften eines Mitgliedstaats vorgesehenen Doppelleistungsbestimmungen nicht für anteilige Leistungen (Abs. 1). Absatz 2 betrifft die Anwendung der Antikumulierungsregelungen bei Leistungen gleicher Art hinsichtlich der autonomen Leistungen. Doppelleistungsbestimmungen gelten nur dann für eine autonome Leistung, wenn es sich um eine Leistung handelt, deren Höhe von der Dauer der zurückgelegten Versicherungs- oder Wohnzeiten unabhängig ist oder es sich um eine Leistung handelt, deren Höhe unter der Berücksichtigung einer fiktiven Zeit bestimmt wird, die als zwischen dem Eintritt des Versicherungsfalles und einem späteren Zeitpunkt zurückgelegt angesehen wird und die zusammentrifft mit einer Leistung gleicher Art, außer wenn zwei oder mehr Mitgliedstaaten ein Abkommen zur Vermeidung einer mehrfachen Berücksichtigung der gleichen fiktiven Zeit geschlossen haben, oder mit einer Leistung nach Buchstabe a). Die genannten Leistungen sind in Anhang IX aufgeführt.

Im Falle des Zusammentreffens von Leistungen unterschiedlicher Art findet Art. 55 VO 883/2004 Anwendung. „Gegenstand dieser Regelung ist das hauptsächliche Koordinierungsproblem bezüglich des in der Regelungskompetenz der Mitgliedstaaten belassenen internationalen Anwendungsbereichs der Antikumulierungsvorschriften, nämlich die mehrfache oder wechselseitige Leistungskürzung oder -entziehung." 29 Die Vorschrift betrifft das Zusammentreffen von Leistungen unterschiedlicher Art und differen-

27 EuGH v. 7.3.1991, Rs. C-10/90 (Masgio), Slg. 1991, S. I-1119.

28 EuGH v. 5.4.1990, Rs. C-109/89 (Pian), Slg. 1990, S. I-1599.

29 Schuler, Kommentar zum Art 55 (Fußn. 3), Rn. 2. 
ziert entsprechend Art. 52 Abs. 1 und 2 danach, ob ein autonomer oder anteiliger Leistungsanspruch besteht. Bei autonomen Leistungen unterschiedlicher Art sind die zu berücksichtigenden Beträge durch die Zahl der an der Kürzung beteiligten Leistungen zu dividieren (Abs. 1 lit. a)). Demgegenüber werden bei anteiligen Leistungen die sonstigen Leistungen im Falle der Mehrfachkürzung nach dem pro-rata-Verhältnis gekürzt (Abs. 1 lit. b)). Treffen eine oder mehrere autonome Leistungen und eine oder mehrere anteilige Leistungen zusammen, so wenden die zuständigen Träger Buchstabe a) auf die autonomen Leistungen und Buchstabe b) auf die anteiligen Leistungen entsprechend an.

Die Teilung der autonomen Leistung ist ausgeschlossen, wenn nach den Vorschriften des zuständigen Mitgliedstaates eine pro-rata-Kürzung vorgesehen ist. 


\title{
Zur grenzüberschreitenden Erbringung von Gesundheitsleistungen
}

\author{
Daniel Eryk Lach
}

A. Einleitende Bemerkungen - Terminologie

I. Grenzüberschreitende Gesundheitsversorgung und Definition der Sachleistungen

II. Begriffe des Versicherungsmitgliedstaates, des zuständigen Trägers und des zuständigen Mitgliedstaates

III. Versicherter und Patient

B. Das Recht auf Leistungen der Gesundheitsversorgung

I. Wohnort in einem anderen als dem zuständigen Mitgliedstaat 203

II. Grenzgänger

III. Aufenthalt außerhalb des zuständigen Mitgliedstaats

1. Die medizinische Notwendigkeit der Leistungen der Gesundheitsversorgung

2. Reisen zur Inanspruchnahme von Sachleistungen Die Genehmigung des Trägers

C. Patientenrechte in der grenzüberschreitenden Gesundheitsversorgung 208

I. Annahmen zur grenzüberschreitenden Gesundheitsversorgung 208

II. Zuständigkeiten der Mitgliedstaaten

1. Zuständigkeiten des Behandlungsmitgliedstaats

2. Zuständigkeiten des Versicherungsmitgliedstaats

III. Erstattung der Kosten

1. Allgemeine Grundsätze - Art. 7 der Richtlinie Nr. 2011/24/EU 214

2. Das Recht des Versicherungsmitgliedstaats auf die Beschränkung der Anwendung von Vorschriften über die Kostenerstattung

3. Das zur Kostenerstattung verpflichtete Subjekt

4. Gesundheitsversorgung, die einer Vorabgenehmigung unterliegen kann

a) Abschließender Katalog der Gesundheitsversorgung mit Vorabgenehmigung

b) Die Beschränkung der Zulässigkeit der Verweigerung der Vorabgenehmigung

D. Schlussbemerkungen 